\title{
Homotopic Transformations of Combinatorial Maps ${ }^{\star}$
}

\author{
Jocelyn Marchadier, Walter G. Kropatsch, and Allan Hanbury \\ Pattern Recognition and Image Processing Group(PRIP) \\ Favoritenstraße 9/1832 A-1040 Wien, Austria \\ jm@prip.tuwien.ac.at
}

\begin{abstract}
In this contribution, we propose the notion of homotopy for both combinatorial maps and weighted combinatorial maps. We also describe transformations that are homotopic in the defined sense. The usefulness of the concept introduced is illustrated using two applications. The first one consists in calculating a skeleton using homotopic transformations of weighted combinatorial maps. The result is a compact combinatorial map describing the structure of the skeleton which may be viewed as a "combinatorial map skeleton". The second application consists in run length encoding of all the regions described by a combinatorial map. Although these demonstrations are defined on combinatorial maps defined on a square grid, the major insights of the paper are independent of the embedding.
\end{abstract}

Keywords: Homotopy, skeletonization, combinatorial map.

\section{Introduction}

Homotopy characterizes, in continuous topology, elastic transformations that preserve certain topological properties, transforming a simple arc into a simple arc for example. The definition of homotopy for digital sets has been proposed [15] in order to characterize transformations of such sets preserving topological properties such as the region inclusion tree, or more generally, equivalence classes of paths. The definition of homotopy of transformations on gray-level images has also been proposed [15,12], as well as on ordered sets [2]. Homotopy is an important concept, as it characterizes topological properties of skeletons, graytone skeletons and watersheds 15121314. Combinatorial maps have been introduced as a code for planar graphs. They have been already used in image analysis to encode topological maps with different embeddings [76]11]. Some transformations of combinatorial maps have been proposed [4.

In this paper, we propose to extend the notion of homotopy to combinatorial maps (section 2), and to weighted combinatorial maps, i.e. combinatorial maps in which a single real number is associated with each dart (section[3), coherently with the classical definitions. The main advantage is in the design of classes

\footnotetext{
* This paper has been supported by the Austrian Science Fund (FWF) under grants P14445-MAT and P14662-INF
} 


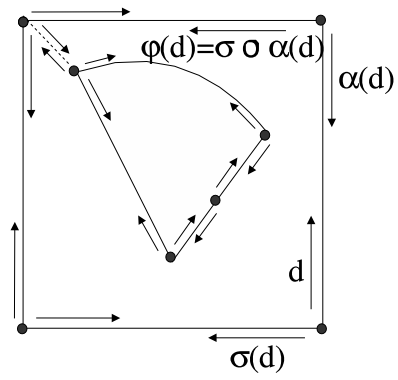

Fig. 1. A combinatorial map

of transformations that have nice topological properties, independently of the embedding of the sets studied. Thus, combinatorial maps with different embeddings can be treated with the same classes of transformations and algorithms such that properties defined independently from their embedding are preserved. Some transformations that are homotopic in the defined sense are also presented. Two applications are presented (section 4), demonstrating the advantages of the proposed definitions and transformations. Defining homotopy on a combinatorial map naturally leads to the definition of a new class of skeletonization algorithm (section 4.1), producing combinatorial map skeletons. The second application, presented in section 4.2 consists of constructing a combinatorial map conveniently encoding horizontal runs.

\section{Combinatorial Maps}

\subsection{Basic Definitions}

Let us review some definitions. A combinatorial map is a triplet $G=(\mathcal{D}, \sigma, \alpha)$ where $\mathcal{D}$ is a set of elements called darts (or half-edges), and $\sigma$ and $\alpha$ are two permutations defined on $\mathcal{D}$ such that $\alpha$ is an involution without fixed point $\left(\forall d \in \mathcal{D}, \alpha^{2}(d)=d\right)$. An example of a combinatorial map is drawn in Fig. [1] Each dart may be viewed as a directed half-edge of an embedded planar graph, and is associated to a vertex. The darts $d$ and $\alpha(d)$ are associated to a unique edge of the drawn planar graph. $\sigma$ defines the arrangement of darts turning counterclockwise around a vertex. A combinatorial map can be seen as a graph with explicit orientation around the vertices.

A combinatorial map may be used to encode a topological map, i.e. a cellular complex of dimension 2 which partitions an orientable surface into a set of vertices (0-cells), a set of arcs (1-cells), and a set of faces (2-cells). Here, the continuous embedding of the underlying cellular complex is assumed, although most of the following results can be also interpreted with embeddings on other topological spaces. The darts of $\mathcal{D}$ may be viewed as cell-tuples $(s, a, f)$ [10], where $s, a$ and $f$ are incident. The orbits $\sigma^{*}(d)$ are bijectively associated to vertices of the represented topological map, the orbits $\alpha^{*}(d)$ are associated to 

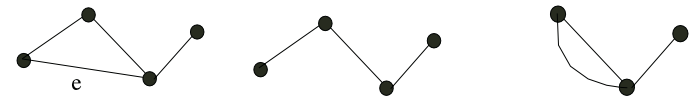

a) Initial map. b) Removal of e. c) Contraction of e.

Fig. 2. Removal and contraction transformations

edges of the topological map, and the orbits $\varphi^{*}(d)$ of the permutation $\varphi=\sigma \circ \alpha$ are associated to the faces of the encoded topological map.

Some topological notions such as loops, bridges, etc, can be defined straightforwardly for combinatorial maps 4]. We recall the following configurations, as they are special cases to be considered in the following text. Let us consider a combinatorial map $G=(\mathcal{D}, \sigma, \alpha)$ and one of its darts $d \in \mathcal{D}$. $d$ is a self loop iff $\alpha(d) \in \sigma^{*}(d)$. $d$ is a bridge iff $\alpha(d) \in \varphi^{*}(d)$. $d$ is pendant iff $\sigma(d)=d$. $d$ is redundant iff $\sigma^{2}(d)=d$.

Paths and loops can also be defined for combinatorial maps. A path of a combinatorial map $G=(\mathcal{D}, \sigma, \alpha)$ is an ordered sequence of darts $P=\left(d_{1}, \ldots, d_{n}\right)$ such that $\forall i \in\{1, \ldots, n-1\}, d_{i+1} \in \sigma^{*}\left(\alpha\left(d_{i}\right)\right)$. The reverse path $\alpha(P)$ of $P$ is defined by $\alpha(P)=\left(\alpha\left(d_{n}\right), \ldots, \alpha\left(d_{1}\right)\right)$. A loop is a path $P=\left(d_{1}, \ldots, d_{n}\right)$ such that $d_{1} \in \sigma^{*}\left(\alpha\left(d_{n}\right)\right)$.

The dual of a combinatorial map $G=(\mathcal{D}, \sigma, \alpha)$ is the combinatorial map $\bar{G}=(\mathcal{D}, \sigma, \varphi)$. It is well defined when the combinatorial map is (path-)connected, i.e. $\forall d, d^{\prime} \in \mathcal{D}, \exists$ a path $P=\left(d_{1}, \ldots, d_{n}\right)$ with $d \in \alpha^{*}\left(d_{1}\right)$ and $d^{\prime} \in \alpha^{*}\left(d_{n}\right)$. In the following text, we will consider only connected combinatorial maps.

The removal of an edge $\alpha^{*}(d)$ removes $d$ and $\alpha(d)$ from the initial combinatorial map. Consider a combinatorial map $G=(\mathcal{D}, \sigma, \alpha)$ and a dart $d \in \mathcal{D}$ with $d$ not being a bridge. The removal of the edge $\alpha^{*}(d)$ creates the sub-map $G \backslash \alpha^{*}(d)=\left(\mathcal{D} \backslash \alpha^{*}(d), \sigma^{\prime}, \alpha\right)$ defined by:

$$
\begin{cases}\sigma^{\prime}\left(d^{\prime}\right)=\sigma\left(d^{\prime}\right) & \text { if } \mathrm{d}^{\prime} \in \mathcal{D} \backslash\left\{\sigma^{-1}(\mathrm{~d}), \sigma^{-1}(\alpha(\mathrm{d}))\right\} \\ \sigma^{\prime}\left(\sigma^{-1}(d)\right)=\sigma(d) & \text { if } \sigma(\mathrm{d}) \neq \alpha(\mathrm{d}) \text { and } \sigma(\alpha(\mathrm{d})) \neq \mathrm{d} \\ \sigma^{\prime}\left(\sigma^{-1}(\alpha(d))\right)=\sigma(\alpha(d)) & \text { if } \sigma(\mathrm{d})=\alpha(\mathrm{d}) \\ \sigma^{\prime}\left(\sigma^{-1}(d)\right)=\sigma(\alpha(d)) & \text { if }\end{cases}
$$

The contraction of an edge $\alpha^{*}(d)$ transforms a combinatorial map $G$ into a combinatorial map $G^{\prime}$ where $d$ and $\alpha(d)$ have been removed from the dual $\overline{G^{\prime}}$. Consider a combinatorial map $G=(\mathcal{D}, \sigma, \alpha)$ and a dart $d \in \mathcal{D}$ with $\alpha^{*}(d)$ not being a self-loop. The contraction of the edge $\alpha^{*}(d)$ creates the sub-map $G^{\prime}$ defined by:

$$
G^{\prime}=G / \alpha^{*}(d)=\overline{\bar{G} \backslash \alpha^{*}(d)}
$$

The two transformations are illustrated in Fig. 2.

\subsection{Homotopic Transformations}

Let us recall that a continuous path is the image of the unit interval by a bijection $f$ into some space $X(f:[0,1] \mapsto X)$. Two continuous paths defined by 
$f:[0,1] \mapsto X$ and $g:[0,1] \mapsto Y$ are said to be homotopic iff there exists a continuous map $H: X \times[0,1] \mapsto Y$ that transforms $f$ into $g: H(x, 0)=f(x)$ and $H(x, 1)=g(x)$. Homotopic transformations are used to define fundamental groups in both continuous and digital topological spaces [1]. The fundamental group of a topological space $X$ is the group formed by all equivalence classes of loops (paths $f$ with $f(0)=f(1)$ ) under the equivalent relation of homotopy.

We introduce the new notion of homotopy on combinatorial maps, derived from the definitions of [15] p.187. However, for combinatorial maps, the dual structure is straightforwardly defined as shown in the previous section, and the definition is simpler.

We say that two paths are equivalent if one can be obtained from the other by a finite sequence of operations of the form:

- if $d_{k}=\alpha\left(d_{k-1}\right)$ replace $\ldots, d_{k-2}, d_{k-1}, d_{k}, d_{k+1} \ldots$ by $\ldots, d_{k-2}, d_{k+1} \ldots$

- or conversely replace $\ldots, d_{k-1}, d_{k} \ldots$ by $\ldots, d_{k-1}, d, \alpha(d), d_{k} \ldots$

Its clear that two equivalent paths must contain the same loops.

Consider the set $\mathcal{G}$ of all combinatorial maps. A mapping $\Phi$ from $\mathcal{G}$ onto itself is said to be homotopic if it transforms a combinatorial map $G$ into a combinatorial map $\Phi(G)$ such that each pair of equivalent path of $G$ is transformed into a pair of equivalent path $\Phi(G)$. As a direct consequence, there must be a bijection between the orbits of $\varphi$ of $G$ and of $\Phi(G)$.

Theorem 1. The contraction of an edge $\alpha^{*}(d)$ is a homotopic transformation.

The proof is straightforward, as the contraction does not either remove nor create a face. This trivial result demonstrates the power of the notion of "homotopy" for combinatorial maps. Moreover, as underlined above, there is a direct interpretation of this notion for continuous topological maps. The contraction operation applied on combinatorial maps will lead to a description of topological maps having the same fundamental group (in the common continuous interpretation). This simple definition is completely equivalent to more complex frameworks [1, and can benefit from previously published results. Moreover, the contraction of pendant darts can be interpreted as the operation of simple point removal, neither of them changing the topology of the described set.

In [9], a dual graph is transformed such that the degree of the surviving vertices is preserved. This rule can be applied to a combinatorial map.

Theorem 2. The contraction of $\alpha^{*}(d)$, where $d$ is a redundant dart, preserves the cardinality of the orbits of surviving darts.

We just give an intuitive idea of the result: Fig. 3 demonstrates the contraction of a redundant dart; as $\left|\sigma^{*}(d)\right|=2$, the contraction of $d$ does not change $\left|\sigma^{*}(\sigma(\alpha(d)))\right|$ and $\left|\sigma^{*}(\alpha(d))\right|$.

Contraction of redundant edges is connectivity preserving. It is a homotopic transformation, as it preserves the number of orbits of $\varphi$ of a combinatorial map. 


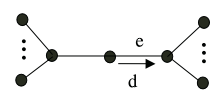

a) Initial map.

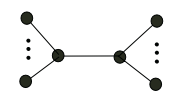

b) Contraction of $d$.

Fig. 3. Contraction of a redundant dart $d$

\section{Weighted Combinatorial Maps}

\subsection{Definitions}

We introduce here new notions related to combinatorial maps whose darts are associated with a single real number.

A weighted combinatorial map is a 4 -tuple $(\mathcal{D}, \sigma, \alpha, w)$ where $(\mathcal{D}, \sigma, \alpha)$ defines a combinatorial map, and $w: \mathcal{D} \rightarrow \mathbb{R}$ is a function defined on $\mathcal{D}$, associating a real number $w(d)$ to each dart $d \in \mathcal{D}$. Weights associated to darts can take any value, depending on the application. We restrict ourselves to the study of a particular class of weighted combinatorial maps, where two opposite darts $d$ and $\alpha(d)$ have opposite weights. We say that a weighted combinatorial map $M=(\mathcal{D}, \sigma, \alpha, w)$ is antisymmetric iff $\forall d \in \mathcal{D}, w(d)=-w(\alpha(d))$.

The following notions interpret weights of darts of a weighted combinatorial map as difference of elevations of connected vertices. An upstream path is a path $P=\left(d_{1}, \ldots, d_{n}\right)$ with only positive weights $\left(\forall i \leq n, w\left(d_{i}\right)>0\right)$. A downstream path is a path $P=\left(d_{1}, \ldots, d_{n}\right)$ with only negative weights $\left(\forall i \leq n, w\left(d_{i}\right)<0\right)$. A plateau path is a path $P=\left(d_{1}, \ldots, d_{n}\right)$ with only null weights $\left(\forall i \leq n, w\left(d_{i}\right)=0\right)$. Since the weights on opposite darts of a weighted combinatorial map can have any value, the reverse of an upstream path is not necessarily a downstream path. However, for an antisymmetric weighted map, this is the case, as stated by the following theorem:

Theorem 3. If a weighted combinatorial map $G=(\mathcal{D}, \sigma, \alpha, w)$ is antisymmetric, then the opposite of every upstream path is a downstream path.

Proof. By definition, the opposite of a path $P=\left(d_{1}, \ldots, d_{n}\right)$ is the path $P^{\prime}=$ $\left(\alpha\left(d_{n}\right), \ldots, \alpha\left(d_{1}\right)\right)$. Suppose that $P$ is upstream, then $\forall i \leq n, w\left(d_{i}\right)>0$. As the map is antisymmetric, we have $\forall i \leq n,-w\left(\alpha\left(d_{i}\right)\right)>0$, and $P^{\prime}$ is downstream.

The removal and the contraction transformations are defined as removal and contraction of the combinatorial map, and do not modify the weights of the remaining darts.

\subsection{Homotopy Revisited}

We define here the concept of homotopy for weighted combinatorial maps, in such a way that it is coherent with the definition of Serra ([15 p. 448). Consider the set $\mathcal{G}$ of all weighted combinatorial maps. A mapping $\Phi$ from $\mathcal{G}$ onto itself is said to be homotopic if it transforms a weighted combinatorial map $G$ into a weighted combinatorial map $\Phi(G)$ such that: 
1. combinatorial maps underlying $G$ and $\Phi(G)$ are homotopic,

2. $\Phi$ preserves upstream and downstream paths.

Two weighted combinatorial maps $G_{1}$ and $G_{2}$ are homotopic iff there exists a homotopic transformation $\Phi$ such that $G_{2}=\Phi\left(G_{1}\right)$.

In the preceding definition, the path preserving condition (condition 2) has to be understood as: any upstream (downstream) path is transformed into an upstream (downstream) path, possibly empty, and no new upstream (downstream) path is created.

The contraction of a dart $d$ of an antisymmetric weighted combinatorial map $G=(\mathcal{D}, \sigma, \alpha, w)$ is path-preserving iff $\sigma^{*}(d)=\{d\}$ ( $d$ is a pendant dart) or $d$ is not a self loop and $w(d) \neq 0$ and $\forall d^{\prime} \in \sigma^{*}(d), w(d) w\left(d^{\prime}\right) \leq 0$ (the weights of $d$ and of any of the darts adjacent to the same vertex have opposite signs). The contraction of path preserving darts is a homotopic transformation, as stated in the following theorem.

Theorem 4. A path-preserving contraction of an antisymmetric weighted combinatorial map $G$ is a homotopic transformation.

Proof. Condition 1. is already proved in theorem 1 Condition 2. holds for the following arguments: If $\sigma^{*}(d)=\{d\}$, then $d$ is a pendant dart, and its contraction does not create new upstream or downstream path. Consider an upstream path $P=\left(d_{1}, \ldots, d_{i-1}, d_{i}, d_{i+1}, \ldots, d_{n}\right)$. By definition, $\forall j \leq n, w\left(d_{j}\right)>$ 0 . By contraction of $d_{i}$, this path will be transformed into the path $P^{\prime}=$ $\left(d_{1}, \ldots, d_{i-1}, d_{i+1}, \ldots, d_{n}\right)$ which is upstream. Suppose that by contraction of a dart $d_{i}$ with $w\left(d_{i}\right) \neq 0$, the non-upstream path $P=\left(d_{1}, \ldots, d_{i-1}, d_{i}, d_{i+1}, \ldots, d_{n}\right)$ is transformed into the upstream path $\left.P^{\prime}=d_{1}, \ldots, d_{i-1}, d_{i+1}, \ldots, d_{n}\right)$. Then, as $P^{\prime}$ is upstream and not $P, \forall j \neq i, w\left(d_{j}\right)>0$ and $w\left(d_{i}\right)<0$. As the combinatorial map is antisymmetric, we have $w\left(\alpha\left(d_{i-1}\right)\right)=-w\left(d_{i-1}\right)<0$. Then $w\left(\alpha\left(d_{i-1}\right)\right) w\left(d_{i}\right)>0$ and the contraction of $d_{i}$ is not path-preserving. This reasoning can also be used for proving that no downstream path is created.

On Fig. 4.a, the contraction of a dart which is not path preserving is illustrated. We can remark that a downstream path which did not exist is created (in bold). On Fig. 4 b, the contraction of the path preserving dart $d$ does not create any upstream nor downstream path.

\section{Applications}

\subsection{Gray-Tone Skeletons}

In this section, we apply the preceding treatments to the computation of skeletons of gray-level images, i.e. thin subsets of the crest network of a gray level image 12. Dual graph contractions invariant to monotonic transformations have been studied in 8 . The framework presented here is different in that it is based on an alternative original graph, and uses the notion of homotopic transformation for combinatorial map discussed above. 


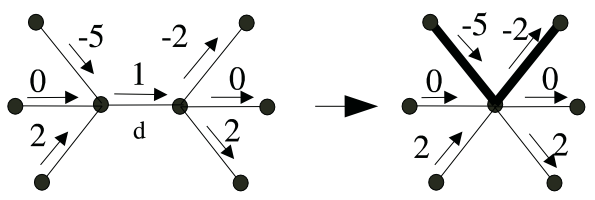

a) Non path preserving contraction.

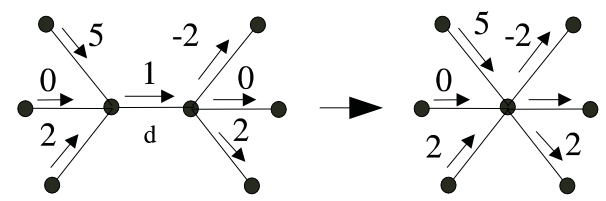

b) Path preserving contraction.

Fig. 4. Contraction of a dart $d$ which is not path preserving

For skeletonisation applications, we start by constructing a weighted combinatorial map on a pixel based scale. Then, by applying some transformations which reduce the number of darts while preserving the homotopy of the combinatorial map until stability is reached, we obtain a compact representation of the skeleton of the original image.

The initial combinatorial map can be obtained from a straightforward algorithm first introduced by M. Pierrot Deseilligny and al. [13]. An image $I$ is defined as a function of digital support $\left[0, x_{\max }\right] \times\left[0, y_{\max }\right]$ to $\mathbb{Z}$. We define $I^{\prime}$ as the image:

$$
I^{\prime}(x, y)=\left(\left(I(x, y) d_{\max }+D(x, y)\right) x_{\max }+x\right) y_{\max }+y
$$

where $D(x, y)$ is the distance of a point $(x, y)$ to the nearest point with lower intensity and $d_{\max }=\max _{(x, y)} D(x, y)$ ( $D$ can easily be related to the classical distance transform of cross-sections of the original gray level images). $I^{\prime}$ assigns to each pixel a unique value. The weighted combinatorial map is built using a simple algorithm based on a local analysis of the 8-neighborhood of each pixel $p$ of $I^{\prime}$. Each $3 \times 3$ neighborhood is decomposed into sets of 4 -connected components, the values of which are greater than the value of the central pixel. We construct the contour map by adding an edge (pair of conjugate darts) that connects the central pixel with the highest valued pixel of each component. The weights being associated to the darts are given by the difference between the end-vertex and the origin-vertex of each dart. The map is obviously antisymmetric. One can demonstrate that the combinatorial map is connected, and that a bijection between the faces ( $\varphi$-orbits) of the combinatorial map and the local minima of $I^{\prime}$ exists 13 .

Homotopic transformations can then be applied in order to simplify the combinatorial map, and to get rid of the undesired edges. For example, darts that are either redundant or pendant, and whose contraction is path-preserving, can be contracted until stability in order to obtain the simplest combinatorial map (kernel of the transformation) describing the crest network of the image. Some 

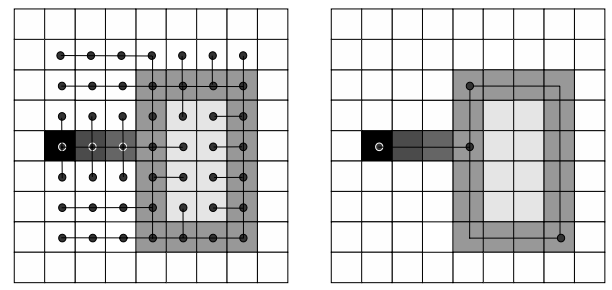

Fig. 5. Skeleton of a weighted combinatorial map

darts describing relevant features can be excluded from the contraction operation in order to preserve these features. This is equivalent to defining anchor points 1314. As a classical example of "anchor darts", we may want to keep pendant darts with negative weights, corresponding to peaks in the image, leading to results very similar to [11]. Fig. 5 shows the results of path-preserving contractions until stability of the redundant darts (on the right), with pendant darts with negative weight as anchor darts (characterizing peaks in the original image).

The recursive contraction of pendant darts is applied first, and then pathpreserving contraction of redundant darts is computed. The algorithm in that order leads to a time complexity linear in the number of edges (two passes on all edges). As the combinatorial map can be constructed within a single pass onto the original image, the complete algorithm is linear in the number of pixels of the original image.

The obtained result is a compact combinatorial map describing the structure of the skeleton in a much more compact way than a raster graph, which may be thought of as a "graph"-skeleton. Hierarchies of combinatorial maps may be defined describing different simplification levels of the underlying graph. The homotopic kernel of the contraction (made up of loops only) may then be thought as the top level of the hierarchy. Other criteria than homotopy such as geometry, etc, may be considered. With the criteria used, the result is independant of the order of the contractions, and the algorithm can be implemented in parallel. The above scheme also works for different types of grid or digital topologies, by giving a proper embedding of the underlying combinatorial maps.

\subsection{Curve-Based Runlength Encoding}

The main idea proposed in this section is to construct a combinatorial map such that in a band defined by two consecutive vertices of the map, no topological events occur (no region appears or disappears, the interior of regions being described by convex domains). For example, in Fig. 6, a simple region described by its boundary is decomposed into two bands (A and B) within which the region is decomposed into connected components which are convex on each horizontal line.

By carefully choosing the weights associated to each dart of the map, we can use the algorithm described in the preceding section in order to compute the 


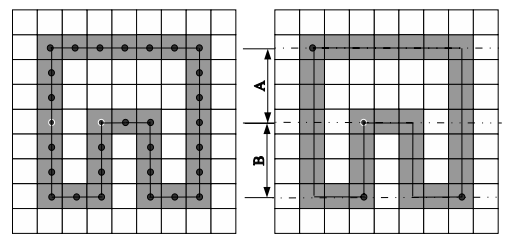

Fig. 6. Curve-based encoding of horizontal runs

combinatorial map. We consider an antisymmetric weighted combinatorial map $G=(\mathcal{D}, \sigma, \alpha, w)$ such that each vertex $\sigma^{*}(d)$ is a point of $\mathbb{Z}^{2}$, each edge $\alpha^{*}(d)$ relates a pair of (4 or 8)-neighbors, and for a dart $d \in \mathcal{D}, w(d)=y^{\prime}-y$ with $y^{\prime}$ and $y$ the vertical coordinate of respectively the vertex $\sigma^{*}(\alpha(d))$ and $\sigma^{*}(d)$.

The algorithm computing the sought combinatorial map performs the contraction until stability of the path-preserving redundant darts. Fig. [6]llustrates the result of such an algorithm. On the left, the initial map is drawn. On the right, the contracted map is represented. The regions $\mathrm{A}$ and $\mathrm{B}$ indicate the rows of the image for which the connected components associated to each region are convex.

The resulting map can be used for filling regions by a simple scan line algorithm which retrieve from the curves horizontal runs [5] describing the interior of regions. The first part of the algorithm is to sort the vertices of the computed map according to the vertical coordinates. Between two vertices, the curves of the map decrease monotonically. Thus, a simple loop can be used to deduce the left and right extremity of a run filling a connected component associated to a region. When a line corresponding to the end of the treated band is reached, the followed curves can be updated according to the topological events that occur on that line (insertion/deletion of followed curves, insertion/deletion of filled connected components).

\section{Conclusion and Perspectives}

In this paper, we have proposed the concept of homotopy for combinatorial maps and weighted combinatorial maps. This leads to defining homotopy between unembedded structures encoding topological maps. The main advantage in doing so is to define homotopic transformations independently of the embedding. Concepts, demonstration, and algorithms proposed are simple. Nenertheless, the proposed concepts lead to results completely analog to the more complex frameworks. This apparent simplicity demonstrates the usefulness of this research.

As an application, we considered the computation of skeletons, for example, for which a compact structure is produced. This naturally extends the concept of skeleton leading to the new concept of "combinatorial map skeletons", i.e. planar embedded graphs which describe homotopic digital or continuous topological structures in the classical sense. The encoding of a combinatorial map whose vertices define bands decomposing the described topological map into 
convex connected components has been proposed in the same framework. Other applications are possible. For example, the computation of hierarchies of skeletons is straightforward. We could also consider the extension of the proposed framework into higher dimensions.

\section{References}

1. Ayala R., Dominguez E., Francés A. R., Quintero A., "Homotopy in digital spaces", Discrete Applied Mathematics 125(1) (2003) 218-228

2. Bertrand G., "New Notions for Discrete Topology", DGCI'99, Lecture Notes in Computer Science no. 1568 (1999) 3-24

3. Braquelaire, J.-P., Brun, L. "Image Segmentation with Topological Maps and Interpixel Representation", Journal of Visual Communication and Image Representation, vol. 9(1) (1998) 62-79

4. Brun L., Kropatsch W. G., "Dual Contraction of Combinatorial Maps", PRIPTR-54, Vienna University of Technology (1999), 37 pages

5. Burge M., Kropatsch W. G., "A Minimal Line Property Preserving Representation of Line Images", Computing, vol. 62, (1999) 355-368

6. Fiorio C., "A topologically Consistent Representation for Image Analysis: the Frontiers Topological Graph", DGCI'96, Lecture Notes in Computer Science no. 1176, (1996) 151-162

7. Gangnet M., Hervé J.-C., Pudet T., Van Tong J.-M., "Incremental Computation of Planar Maps", SIGGRAPH Proc., Computer Graphics, vol. 23(3) (1989) 345-354

8. Glantz R., Englert R., "Dual Image Graph Contractions Invariant to Monotonic Transformations of Image Intensity", In Proc. of the 2nd Int. IAPR Workshop on Graph-based Representation, 1999.

9. Kropatsch W.G., "Property Preserving Hierarchical Graph Transformations", Advances in Visual Form Analysis, C. Arcelli, L. Cordella and G. Sanniti di Baja Eds (1997) 340-349

10. Lienhardt P., "Topological Models for boundary representation: a comparison with n-dimensional generalized maps", Computer Aided Design vol. 23(1) (1991) 59-82

11. Marchadier J., Arquès D., Michelin M., "Thinning Grayscale Well-Composed Images: A New Approach for Topological Coherent Image Segmentation", DGCI'02, Lecture Notes in Computer Science no. 2301 (2002) 360-371

12. Meyer F., "Skeletons and Perceptual Graphs", Signal Processing, vol. 16 (1989) 335-363

13. Pierrot Deseilligny, M., Stamon, G., Suen, C., "Veinerization: A New Shape Description for Flexible Skeletonization", IEEE Trans. on PAMI, vol. 20(5) (1998) $505-521$

14. Ranwez V., Soille P., "Order independent homotopic thinning for binary and grey tone anchored skeletons", Pattern Recognition Letters, vol. 23 (2002) 687-702

15. Serra J., "Image Analysis and Mathematical Morphology", Academic Press, London, 1982, 610 pages. 